\title{
Clinical, histopathological, and dermoscopic features of melanotic macules of the glans penis
}

\section{Hiroki Furukawa', Toshiyuki Ozawa', Junko Sowa-Osako', Hiroshi Sakai', Takashi Hashimoto', Daisuke Tsuruta ${ }^{1}$}

${ }^{1}$ Department of Dermatology, Osaka City University Graduate School of Medicine, Osaka 545-8585, Japan, ${ }^{2}$ Department of Dermatology, Osaka Police Hospital, Osaka 543-0035, Japan

Corresponding author: Prof. Takashi Hashimoto, MD, E-mail: hashyt@gmail.com

Sir,

Melanotic macules of the glans penis are benign pigmented lesions that may clinically mimic melanomas [1-3]. Herein, we report a case of this condition with clinicopathological and dermoscopic findings.

A 37-year-old Japanese male presented himself with a five-year history of pigmented lesions on the glans penis. The patient underwent circumcision for the treatment of phimosis seven years ago but had no family history of melanoma.

A physical examination revealed serpiginous black and brown macules on the glans penis (Fig. la). Our differential diagnoses included melanotic macules, melanocytic nevus, pigmented lichen planus, and melanoma. A dermoscopic examination revealed multiple patterns, including a light brown structureless zone in the center with dark brown circles and dots and lines at the periphery (Fig. lb). The lesion was generally globular and arranged asymmetrically. Sharp whitish lines were seen along the skin grooves, which excluded melanoma. A histopathological examination of a punch biopsy from the dark brown macule showed abundant melanin in basal keratinocytes without increase in melanocytes (Fig. 1c). There was no evidence of malignancy. Based on these features, the diagnosis of melanotic macules of the glans penis was reached.

Dermoscopy is a useful method for the accurate diagnosis of pigmented skin lesions, particularly melanomas [1]. The number-needed-to-excise (NNE) value is calculated by dividing the total number of lesions excised by the number of malignant melanomas. A study of over 300,000 cases of melanomas and melanocytic nevi demonstrated that NNEs were significantly lower in "specialized clinical settings" than in "non-specialized settings," and improved over time only in "specialized clinical settings" - which might have been contributed to by more frequent use of dermoscopy [2].

Pigmented lesions of the genital region are relatively uncommon [1-3]. Particularly, there have been only a few dermoscopic studies of pigmented genital lesions in males [4], whereas vulvar melanotic macules have been more frequently studied with dermoscopy [5]. Cengiz et al. reported that melanosis was the most common pigmented lesion in the genital region, and that the glans penis was the most frequent location for pigmented lesions in males [4]. Half of the cases of melanosis of the genital region had multifocal lesions, of which approximately $70 \%$ showed two to three colors and half showed a globular pattern [4].

Our patient had a multifocal two-colored pigmented lesion with a globular pattern, which is considered a common dermoscopic feature of melanotic macules of the glans penis.

In conclusion, dermoscopy is a useful technique for the differentiation of melanotic macules of the glans penis from other pigmented lesions in the genital region.

\section{ACKNOWLEDGMENTS}

We would like to thank Dr. Libby Cone for the critical reading of the manuscript.

\footnotetext{
How to cite this article: Furukawa H, Ozawa T, Sowa-Osako J, Sakai H, Hashimoto T, Tsuruta D. Clinical, histopathological, and dermoscopic features of melanotic macules of the glans penis. Our Dermatol Online. 2021;12(1):86-87.

Submission: 20.11.2020; Acceptance: 02.12.2020

DOI: 10.7241/ourd.20211.25
} 

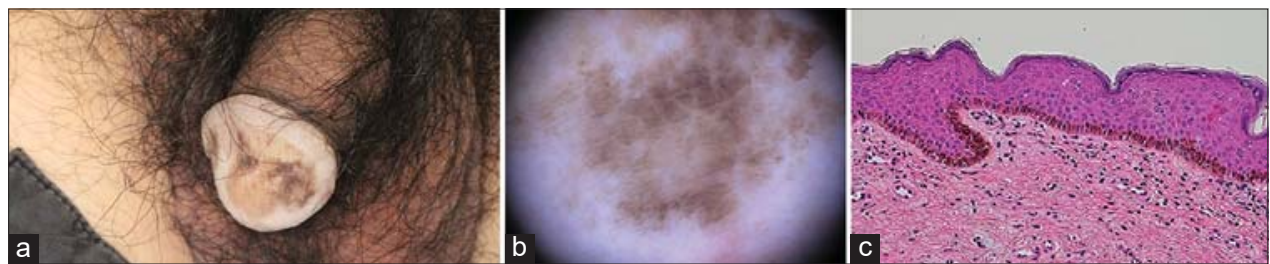

Figure 1: Clinical, dermoscopic, and histopathological features in our case. (a) Clinical features on the glans penis. (b) Dermoscopic findings. (c) Histopathological features (H\&E, 400x).

\section{Consent}

The examination of the patient was conducted according to the principles of the Declaration of Helsinki.

The authors certify that they have obtained all appropriate patient consent forms, in which the patients gave their consent for images and other clinical information to be included in the journal. The patients understand that their names and initials will not be published and due effort will be made to conceal their identity, but that anonymity cannot be guaranteed.

\section{REFERENCES}

1. Soyer HP, Argenziano G, Chimenti S, Ruocco V. Dermoscopy of pigmented skin lesions. Eur J Dermatol. 2001;11:270-6; quiz 277.
2. Argenziano G, Cerroni L, Zalaudek I, Staibano S, HofmannWellenhof R, Arpaia N, et al. Accuracy in melanoma detection: A 10-year multicenter survey. J Am Acad Dermatol. 2012;67:54-9.

3. Betti R, Menni S, Crosti C. Melanoma of the glans penis. Eur J Dermatol. 2005;15:113-5.

4. Cengiz FP, Emiroglu N, Wellenhof RH. Dermoscopic and clinical features of pigmented skin lesions of the genital area. An Bras Dermatol. 2015;90:178-83.

5. Ronger-Savle S, Julien V, Duru G, Raudrant D, Dalle S, Thomas L. Features of pigmented vulval lesions on dermoscopy. Br J Dermatol. 2011;164:54-61.

Copyright by Hiroki Furukawa, et al. This is an open-access article distributed under the terms of the Creative Commons Attribution License, which permits unrestricted use, distribution, and reproduction in any medium, provided the original author and source are credited.

Source of Support: Nil, Conflict of Interest: None declared. 